\title{
Quelle perception de la responsabilité sociale des entreprises pour les gestionnaires de fonds en Suisse?
}

\begin{abstract}
Résumé
Nous étudions le lien entre l'engagement durable et social des entreprises (RSE) et leur performance, tout d'abord par une analyse de la recherche dans le domaine, suivie d'une étude qualitative de ce lien sous la forme d'interview de gestionnaires de fonds en Suisse.

Nous trouvons que le lien positif entre la performance des entreprises et leur engagement RSE n'est prouvé et perçu que dans certain contextes. D'autre part, il semblerait que les investisseurs n'attendent pas forcément une performance plus importante, mais une neutralité, c'est-à-dire que la RSE ne nuise pas à la performance. Ils seraient ainsi prêts à investir de préférence dans les entreprises qui créent le plus de valeur dans les objectifs non financiers. Un autre résultat important est que la RSE serait difficile à évaluer de nos jours par les investisseurs « traditionnels », principalement due au manque d'information récente et pertinente sur la RSE des entreprises. La nécessité de faire leur propre recherche crée une certaine barrière, un coût additionnel, que tous les investisseurs ne veulent pas dépasser ou assumer compte tenu de l'absence de gain attendu sur la performance de leurs portefeuilles.
\end{abstract}

\section{Mot clés}

RSE, ESG, Performance Financière, Développement Durable, Management Durable, Gestion de portefeuille, Fonds

\begin{abstract}
We study the link between the sustainable and social activities of a firm (know as Corporate Social Responsibility CSR ) and its financial performance. We first review and analyze the existing research on the topic, followed by a qualitative study of this relationship consisting of interviews of fund managers in Switzerland.

We find in the literature that a positive relationship is perceived between the commitment to CSR of companies and their performance, but overall scientific evidence is still lacking, as positive results are only achieved in some specific contexts. On the other hand, it appears from our interview that investors may not expect a higher performance when investing in high CSR firms, but a neutral relationship. In other words, they want the proof that engaging in CSR is not detrimental to the performance of the firm. In that case, they are willing to invest preferably in companies that create the most value in non-financial objectives.

Another important result is that investors cannot asses the CSR the firm precisely due to a lack of recent and relevant information on companies' CSR activities .The need to do their own research for CSR evaluation could be a barrier for sustainable investment, as investors would spend extra time to ensure of a firm sustainability for a neutral impact on their portfolios gain.
\end{abstract}

\section{Key words :}

CSR, ESG, Financial Performance, Sustainable Development, Sustainable Management, Portfolio Management, Funds,SRI 


\section{Introduction}

Maîtriser les risques environnementaux, mettre en place des processus d'écoconception, développer des produits dits « verts », améliorer la qualité de vie au travail et lutter contre toutes formes de discrimination, communiquer des indicateurs " extra-financiers » sont devenus des actes déterminant de l'engagement et de l'intériorisation institutionnelle de la responsabilité sociétale des entreprises (RSE). Mais, le fait de «s'engager à être responsable ou développer une politique RSE », est-ce une contrainte ou un levier de performance pour les entreprises?

Les études scientifiques empiriques semblent indiquer un lien positif entre les efforts en responsabilité sociale des entreprises, en anglais " corporate social responsability » et leur performance financière. Cependant, elles font parfois de preuve de faiblesses méthodologiques ou d'un contexte limité. Il devient ainsi nécessaire de confirmer les résultats de ces études, qui dépendent largement de l'environnement économique, et d'explorer le sens de la causalité entre performance et responsabilité sociale, lequel est généralement ignoré (Scholtens, 2008).En effet, est-ce bien l'engagement social de l'entreprise qui crée de la valeur, ou bien les entreprises qui génèrent le plus de bénéfices sont-elles celles qui ont les moyens de cet engagement?

Un autre point crucial afin d'appréhender l'impact de cette relation sur la gestion des firmes est de mieux comprendre la perception de ces liens par les investisseurs. En postulant que la RSE créée de la valeur, si cette relation n'est pas perçue correctement par les investisseurs, elle ne sera pas reflétée directement dans le prix des actions des entreprises. A l'inverse, cela pourrait offrir des opportunités pour l'investisseur conscient de ce lien. Il est donc important d'analyser l'avis des praticiens, gestionnaires et investisseur professionnels, pour mieux comprendre leur vision de l'impact de la RSE sur la valeur des actions.

\section{Cadres théorique et méthodologique}

\subsection{La relation entre performance financière et performance non financière}

Selon la théorie des parties prenantes, une meilleure satisfaction de toutes les parties prenantes implique une meilleure maîtrise des coûts de l'entreprise et se traduit par une performance financière supérieure (Waddock et Graves, 1997). L'entreprise est ainsi tenue à ne plus s'intéresser uniquement à sa performance financière, mais également à sa performance sociétale (Gössling, 2003). Au contraire, selon Friedman (1970), la responsabilité sociale des entreprises (RSE) conduit à une expropriation des profits des actionnaires, au bénéfice de la collectivité. De plus, la non-maximisation du profit pour la firme implique une perte d'efficacité pour la société dans son ensemble. Pour éclairer ce débat, de nombreuses études empiriques se sont alors intéressées au lien entre RSE (au travers de la corporate social performance, notée CSP) et performance financière (CFP).

Plusieurs études dont les synthèses de Wu (2006), Maron (2006), Margolis \& Walsh (2003) montrent que la relation entre CSR (ou RSE en français) et performance financière semble positive. Toutefois, leurs résultats sont contestables et contestés car ils dépendent principalement de la nature des données collectées. En effet, ces études reposent pour la plupart sur au moins un des facteurs limitatifs suivants: 
* le marché américain. Les conclusions ne sont donc pas transposables aux firmes européennes compte tenu de leur environnement,

* des données trop anciennes, avant 2000 et la publication des premières «Guidelines du GRI», lesquelles visent à inciter les firmes au niveau mondial à communiquer des informations plus standardisées, précises et comparables en matière de développement durable,

* des données récentes mais avec peu d'indicateurs de CSR et un historique limité (entre 2 et 5 ans au maximum),

* des méthodologies quantitatives fondées sur des données agrégées (note globale de la firme sur la gouvernance par exemple), ou des indicateurs choisis en fonction de leur disponibilité. Par exemple, un des piliers du développement durable, la gouvernance est appréhendée dans la pluparts des articles scientifiques uniquement au travers du nombre d'administrateurs indépendants car cette information est disponible dans les bases de données financières classiques, sans s'intéresser aux liens que peuvent avoir entre eux les administrateurs, à leurs compétences, ou encore à leur rémunération,

* des méthodologies qualitatives d'analyse de discours, utilisant les rapports annuels ou des interviews avec les manageurs sans recouper les informations qui sont purement déclaratives (la triangulation des données est pourtant une condition minimale de ce type d'étude).

De plus, la pluparts des travaux sur la CSR portent sur des recherches antérieures à l'année 2000. Or comme le précisent Cohen and Winn (2007) et Schubert and Lang (2005), même si le rapport Brundtland est considéré comme le point culminant dans la prise de conscience de la CSR aussi bien pour les firmes, les parties prenantes que les analystes financiers, ce n'est qu'au début des années 2000, que le développement durable s'installe comme un enjeu majeur du gouvernement d'entreprise. Les firmes ont dû prendre en compte le changement observé dans les systèmes de valeurs de leurs actionnaires, employés, clients etc., en se prêtant à la mise en œuvre des objectifs sociaux et environnementaux de toutes ses parties prenantes.

Dès lors, les méta-analyses de Margolis, Elfenbein \& Walsh (2007), mais surtout celle de van Beurden and Gössling (2008) semblent plus pertinentes dans la mesure où elles sont essentiellement axées sur des travaux réalisés entre 1990 et 2007 et prennent ainsi en considération ces changements de valeurs sociétales. Ils démontrent que la majorité des études ont mis en évidence une relation positive entre la CSP et la CFP. Si dans ces différentes études, les résultats sont souvent mitigés, c'est essentiellement en raison des mesures de performance choisies et de problèmes méthodologiques, notamment parce que les performances sociétales et financières sont endogènes. La performance sociétale des firmes est appréhendée généralement au travers d'indices de pollution, de réputation, d'agence de notation sociétale telle que KLD, de l'analyse du contenu de leur rapport annuel (analyse de discours), de leurs activités philanthropiques, ou encore de leur inclusion dans un indice boursier dit «socialement responsable» comme le DSI 400 pour les Etats-Unis (Decock-Good, 1991). Certains chercheurs tel que Belu (2009) propose même de nouveaux indices, à partir de la méthode DEA, traduisant une mesure de l'engagement des firmes dans les pratiques de développement durable (ou durabilité). La performance financière comprend des mesures issues de la comptabilité (par exemple: retour sur investissement, ou encore rentabilité des actifs) et d'autres de nature boursière (prix ou rendement des actions). La relation est beaucoup plus significative pour la performance sociétale avec les indices de réputation, et pour la performance financière avec des mesures comptables. Outre l'intensité de la relation plus importante, les mesures issues de la comptabilité présentent l'avantage de fournir une mesure plus pertinente de la performance économique de l'entreprise. Le seul inconvénient provient du fait qu'elles sont plus sujettes à des manipulations managériales (McGuire et al., 1988). Ces mesures, qui sont à la base des 
différences observées dans les résultats, nous amènent à nous interroger sur la notion de performance à long terme des firmes, que la performance financière seule ne peut capter.

Outre ces mesures, l'industrie à laquelle une firme appartient doit être prise en considération dans l'intensité de la relation positive entre durabilité et performance. En effet, les firmes doivent faire face à des préoccupations sociales, environnementales et financières bien distinctes selon leur secteur d'activité. Un établissement bancaire n'aura pas les mêmes préoccupations en termes de développement durable qu'une société pétrochimique. Or, la plupart des études réalisées couvrent plusieurs industries et tendent à masquer les effets d'une industrie spécifique. Comme le propose Chand (2006), ces études sur le lien entre CSP et CFP devraient se focaliser sur une industrie unique.

Cette relation positive entre durabilité et performance est également de plus en plus partagée par les professionnels, comme le montre l'étude de McKinsey-BCCC (2008) dans laquelle deux-tiers des managers et trois-quarts des professionnels de l'investissement interrogés aux USA pensent que la RSE crée de la valeur pour les actionnaires, dans un contexte économique stable.

Par ailleurs, les revues professionnelles et journaux font apparaître trois constats :

1. Les entreprises (leurs présidents) croient que leurs compagnies seraient plus fortement évaluées si les investisseurs comprenaient mieux la performance non financière de leur firme (NFP);

2. Les investisseurs croient qu'ils pourraient mieux évaluer la performance future des firmes si ces dernières étaient disposées à partager plus d'information sur les indicateurs clés de gestion, en particulier non financiers (par exemple leadership, stratégie, développement personnel, service à la clientèle, innovation produit, gestion des risques, réputation) ;

3. Il y a une corrélation forte entre les indicateurs clés de gestion et le management des enjeux environnementaux, sociaux et de gouvernance.

Les études académiques et ces observations montrent qu'au-delà de la mesure des effets du management des enjeux environnementaux, sociaux et de gouvernance (ESG) sur la performance des entreprises, un second problème, celui de leur perception par les investisseurs vient se greffer. Il repose sur les asymétries d'information entre les investisseurs et les sociétés. Certaines causes de ces asymétries d'information ont été identifiées dans la littérature ( $\mathrm{Wu}$, 2006) au niveau :

* Des entreprises :

- manque d'expertise dans les enjeux de la performance non financière de leur firme (NFP) et réticence à discuter d'éléments pourtant non étroitement liés à leur stratégie opérationnelle immédiate,

- doute sur la robustesse de la NFP et des méthodologies internes pour la fournir,

- manque de confiance dans la capacité à gérer la NFP pour fournir des données prévisionnelles

;

- Scepticisme des investisseurs dans la NFP ;

- inquiétude au sujet de l'impact sur la crédibilité personnelle qui résulte de la promotion de messages financiers non orientés sur le cœur de métier.

* Des investisseurs :

- lien causal entre la NFP et la performance financière non évident;

- manque d'études inter-sociétés ou sectorielles robustes sur la comparaison de NFP ; 
- expertise limitée sur l'évaluation de la NFP qui influence directement les compétences en stratégie et leadership ;

- protection de la propriété des outils analytiques de mesure de la NFP créant un manque de transparence dans les méthodologies d'évaluation de la NFP ;

- importance relative des enjeux de la NFP qui oscillent entre la gestion des risques et les normes imposées par le législateur et les régulateurs.

C'est la raison pour laquelle, nous nous sommes intéressés la perception de la performance non financière de la firme (NFP), au travers de la CSR par les investisseurs.

La littérature financière identifie deux éléments fondamentaux dans les décisions d'investissements (Albouy, 2005) :

* la rationalité des investisseurs. En effet, toute prise de décision d'investissement passe par un processus fondé sur l'intuition et/ou le raisonnement. Les investisseurs convaincus des bienfaits du développement durable (DD) ont peut-être plus recours à leur intuition (sur le DD) que les autres investisseurs qui utilisent davantage les raisonnements financiers traditionnels,

* l'horizon de gestion et la performance des entreprises. L'actionnaire qui se place dans une perspective de court terme aura vraisemblablement un comportement différent à celui qu'il aurait s'il investissait à long terme. Un investisseur ayant un horizon à long terme analysera les grandes tendances de l'entreprise (analyse fondamentale de sa performance actuelle et future) et de son secteur car il sait qu'à long terme une firme bien gérée doit créer de la valeur. Tandis qu'un investisseur à court terme sera plus tenté « de faire des coups », et s'intéresser à tous les éléments qui peuvent faire varier les cours rapidement dont les rumeurs.

La diversité des horizons temporels implique donc autant de stratégies d'investissement qui se répercutent différemment sur les dimensions de la CSP (Johnson et Greening, 1999). Les investisseurs éthiques sont-ils à la recherche de gains financiers accrus par des critères de sélections spécifiques ou désirent-ils effectuer des placements à long terme dans le but d'agir sur le comportement des entreprises? Dans ce dernier cas, ils sont susceptibles d'accorder une importance plus grande aux informations sociétales et donc d'influencer leur diffusion.

On peut donc se demander si les investisseurs et gestionnaires de fonds ISR ont la même rationalité que les autres investisseurs, ou s'agit-il seulement d'une autre vision de l'horizon de gestion et de la performance des firmes?

Cette question de la rationalité a surtout été abordée au travers des croyances des investisseurs éthiques. Lewis et Mackenzie (1999) postulent que les décisions des acteurs ne sont pas uniquement motivées par la satisfaction des intérêts privés mais également par des considérations d'ordre moral. Ils considèrent que l'investissement éthique illustre concrètement ce postulat. La question qui se pose est donc de savoir en quoi ces investisseurs se différencient des investisseurs traditionnels ? Face au choix d'un investissement financier, leurs croyances impliquent-elles qu'ils n'effectueront pas clairement des choix « rationnels » au sens de Simon (1955) ? En d'autres termes sont-ils prêts à sacrifier leurs gains financiers pour détenir des actifs éthiques?

Les résultats de plusieurs études montrent que lorsque la performance financière des fonds éthiques est inférieure à celle des fonds classiques, et que les investisseurs " responsables » décident alors, en majorité, de conserver leurs parts dans ces mêmes fonds (Lewis et Mackenzie, 2000a). Ils élaborent des options de choix incompatibles avec celles d'un investisseur traditionnel car ne permettant pas d'atteindre les paliers de satisfaction résultant d'une rationalité «procédurale». 
L'apport des recherches dans ce domaine réside principalement dans le fait qu'elles remettent en cause le postulat de l'agent économique rationnel.

Par ailleurs, les travaux de Webley et al. (2001) apportent des éléments cruciaux afin de mieux comprendre les caractéristiques psychologiques des investisseurs éthiques, leurs motivations et leurs comportements.

Le résultat principal est que l'investissement éthique relève non pas de la recherche de gains financiers mais d'une idéologie et d'une identité particulière. Cette conclusion va à l'encontre des résultats de Mackenzie et Lewis (1999) mais est convergente avec ceux de Lewis et Mackenzie (2000b). De cette étude sont issus d'autres résultats surprenants. Ainsi, les investisseurs éthiques continuent à investir dans un fonds éthique lorsque ses performances financières sont décevantes.

Ainsi, une réponse claire est apportée à la question de la recherche des gains financiers : elle ne ferait pas partie de leurs préoccupations. Mais on peut se demander si la réponse n'est contingente à la méthodologie employée.

Ces recherches sont très importantes car elles constituent une synthèse des études précédentes. En effet, même si leur méthodologie diffère, leur objet de recherche est défini à partir des conclusions tirées des travaux précédents et notamment de l'identification d'un paradoxe dans le comportement des investisseurs éthiques. Les résultats clarifient les éléments de réponse apportés jusqu'à présent en donnant des précisions sur les caractéristiques psychologiques des investisseurs éthiques.

Les travaux de Bénabou et Tirole (2006) offrent également un éclairage intéressant sur la demande pour les fonds ISR. En effet, ils indiquent que cette demande peut émaner de plusieurs sources. D'abord de l'altruisme pur. "D'une certaine manière, nous aspirons tous à faire le bien et à nous rendre utiles, souligne Jean Tirole". Ils montrent aussi que les incitations financières ne peuvent être négligées, les investisseurs dans les fonds ISR attendant une performance financière décente. Ces travaux montrent enfin qu'investir dans l'ISR constitue un moyen de gagner du prestige social.

Ils indiquent donc que les fonds ISR pourraient attiser la demande pour leurs produits financiers en s'appuyant sur le désir d'image de soi des investisseurs via par exemple des campagnes de communication adaptées ou une conception de fonds appropriée.

En s'intéressant aux processus cognitifs, ce courant émergeant de la littérature financière commence à proposer une véritable analyse de la rationalité des investisseurs éthiques. Cette analyse permet d'appréhender ces derniers non pas comme des agents irrationnels mais comme des agents possédant une rationalité spécifique. Toutefois, les fondements théoriques sont absents de la plupart de ces articles. Cette absence, caractéristique d'un champ de recherche émergent, rend délicate l'analyse des résultats et leur généralisation.

C'est pourquoi nous avons choisi une approche plus qualitative afin de comprendre le comportement et les choix des gestionnaires de fonds face aux critères ESG.

\subsection{Méthodologie}

Afin de comprendre le comportement et les choix des gestionnaires de fonds face aux critères ESG, nous avons développé une approche plus qualitative. Notre étude est basée sur des entretiens (questionnaires semi-directifs) de huit gestionnaires de fortune à Genève et Zurich. 
Les entretiens semi-directifs nécessitent la constitution d'un guide thématique formalisé et la planification de stratégies d'écoute et d'intervention. Le guide d'entretien structure l'interrogation mais ne dirige pas le discours. Dans notre cas, il est composé des thèmes suivants :

- l'engagement des entreprises suisses envers le développement durable,

- la manière dont les Asset Managers utilisent des critères liés au développement durable dans leur processus d'investissement,

- le lien, positif ou négatif, qu'ils perçoivent entre la performance d'une entreprise et ses effort en terme de développement durable, et ce pour chacune des quatre catégories : écologie, gouvernance d'entreprise, éthique ou sociétal, sociale,

- les données et fournisseurs qu'ils utilisent pour juger les entreprises selon des critères ESG et la pertinence de ces informations,

- Les motivations ou incitations à introduire des critères non financiers dans la gestion des investissements.

L'analyse de leur discours nous a permis d'appréhender comment ils perçoivent les politique de développement durable des firmes, et intègrent des critères ESG dans leurs choix de portefeuille en fonction des demandes de leurs clients. On a ainsi pu repérer certains de leurs principes de gestion de portefeuille et valeurs sous-jacentes dont nous traçons les contours dans la section suivante.

\section{Analyse des résultats et discussion}

\subsection{La gestion de fonds ISR en Europe et en Suisse}

L'Investissement Socialement Responsable (ISR) a connu une popularité croissante au cours de la dernière décennie. On peut définir l'ISR comme l'introduction de critères liés au développement durable dans les décisions d'investissement, par opposition à l'investissement classique qui se concentre uniquement sur des paramètres financiers. Les critères du développement durable sont habituellement regroupé en trois thèmes : Environnement, Social / Sociétal et Gouvernance d'entreprise (ESG).L'environnement semble être le thème prépondérant, suivi par la gouvernance d'entreprise, le social, et enfin plus récemment l'aspect sociétal ou communautaire. ${ }^{1}$

Selon les rapports bi-annuels US SIF (Sustainable investment forum) et EURO-SIF la masse sous gestion ISR aux Etats-Unis et en Europe serait en forte augmentation. Elle serait ansi passé de 2,1 milliards de dollars en 1999 à $\$ 3,7$ milliards en 2012 pour les états Unis, et de $€ 1,7$ milliards en 2005à $€ 11,7$ milliards en 2011(chiffre comprenant les exclusions basée sur des normes depuis 2009 .

Cet augmentation de l'ISR provient surtout des investisseurs institutionnels (94\%), les fonds publics étant soumis à la pression morale des communautés et des législateurs en matière de développement durable. La popularité de l'investissement responsable est devenue encore plus

\footnotetext{
${ }^{1}$ CSRHub user average Priorities : Community 2.6, Employees 2.8, Environment 3.7, Governance 2.9 http://www.csrhub.com/csrhub/
} 
importante suite à la crise financière de 2007 qui brisé la confiance des investisseurs dans les marchés financiers et les modes d'investissement traditionnels, et entrainé de nouvelles politiques et règles de la part des gouvernement et organes de régulation des marché. L'ISR, en plus d'apporter aux investisseurs une certaine satisfaction morale s'est avéré être un investissement plus stable en période de crise boursière, suscitant un intérêt accrus pour ce type d'approche de portefeuille.

Regardons plus en détail les stratégies qui se rattachent à l'investissement socialement responsable. La forme la plus ancienne de l'ISR est l'exclusion de certains secteurs ou activités jugées non conforme à la morale ou aux dogmes d'une religion, comme les armes, l'alcool, le tabac, etc .. Ce type de stratégie existe depuis le $18^{\text {ème }}$ siècle mais il est maintenant complété par maintenant les exclusions fondées sur des normes internationales récentes. Dans son ensemble, l'exclusion reste la forme la plus appliquée de stratégie ISR et représente toujours plus de la moitié des investissement l'ISR en Europe, avec environ 33\% pour l'exclusion par activité et $20 \%$ pour l'exclusion normative. ${ }^{2}$

Les autres stratégies de la forme moderne de l'ISR ne fonctionnent pas par exclusion des moutons noirs mais au contraire par la sélection active d'entreprises répondant positivement ux critères du développement durable. Environ 28\% de l'ISR en Europe consiste ainsi à integrer des critères ESG en plus des critères financier dans la sélection active d'entreprises. Quant à l'approche « best- in-class », elle favorise les entreprises les mieux notées selon les critères ESG parmi les autres entreprises du même secteur, mais ne représente que $2 \%$ des stratégies. Finalement, dans les stratégies les plus engagées envers le développement durable on notera la création de fonds thématiques, ou l'utilisation du droits des actionnaires pour orienter la stratégie d'une d'entreprise vers l'ESG, stratégie de plus en populaire qui représente $17 \%$ de l'ISR.

\begin{tabular}{|l|l|l|}
\hline \multirow{2}{*}{$\begin{array}{l}\text { Type } \\
\text { Segative }\end{array}$} & Strategies & Definition \\
\hline \multirow{3}{*}{$\begin{array}{l}\text { Positive } \\
\text { screening }\end{array}$} & $\begin{array}{l}\text { Exclusion } \\
\text { screening }\end{array}$ & Exclusion of certain sectors such as weapons etc. \\
\cline { 2 - 3 } & $\begin{array}{l}\text { ESG } \\
\text { Integration }\end{array}$ & Integration of ESG criteria to classic Financial analysis \\
\cline { 2 - 3 } & Best-in-Class & Selection or Weighting of stocks according to ESG criteria \\
\hline \multirow{2}{*}{$\begin{array}{l}\text { Active } \\
\text { Investment }\end{array}$} & $\begin{array}{l}\text { Engagement } \\
\text { Voting }\end{array}$ & $\begin{array}{l}\text { Funds with a theme focused on Sustainability, such as Green energy, } \\
\text { Health, etc. }\end{array}$ \\
\cline { 2 - 3 } & $\begin{array}{l}\text { Impact } \\
\text { Investment }\end{array}$ & $\begin{array}{l}\text { Investing to create an ESG impact such as microfinance, local business } \\
\text { funds, etc... }\end{array}$ \\
\hline
\end{tabular}

Si on se penche sur la Suisse en particulier, l'exclusion reste une des stratégies ISR les plus présentes, notamment par l'exclusion des armes à fragmentation, ce qui a été confirmé dans nos entretiens. La stratégie best -in-class est également très présente et les fonds thématiques viennent en troisième.

\footnotetext{
${ }^{2}$ EURO SIF Europe SRI Study 2012 - http://www.eurosif.org/research/eurosif-sri-study/sri-study-2012
} 
Notre premier thème concerne l'engagement des entreprises suisses envers le développement durable. Dans l'ensemble, les praticiens perçoivent les entreprises suisses comme très engagés, en particulier dans l'environnement, et visant à être les championnes du développement durable au cours des dix à quinze prochaines années. Cela n'aurais pas toujours été le cas dans le passé, en raison notamment d'une réglementation plus permissive. Nos gestionnaires ont noté que l'engagement des entreprises est très variable d'une entreprise à l'autre. Dans l'ensemble, les entreprises ayant une exposition directe aux consommateurs comme Nestlé sont plus actives dans le développement durable et sont capables de gérer leur risque de réputation de manière très active.

Notre deuxième sujet concerne la manière dont les Asset Managers utilisent des critères liés au développement durable dans leur processus d'investissement. Il faut noter que nos gestionnaires ne gèrent pas des fonds thématiques ESG, mais des fonds en actions classiques, suisses ou internationaux. Fait intéressant, la plupart d'entre eux ont déjà mis en place une liste d'exclusion des armes controversées, comme les mines antipersonnel, les bombes à fragmentation, etc.. depuis plusieurs années. Ils admettent que ce genre de filtre assez facile à mettre en œuvre, en particulier pour la gestion quantitative. Toutefois, s'ils sont prêts à exclure quelques entreprises provenant d'une liste, ils n'excluraient jamais un secteur entier, car ils estiment que cela pourrait nuire à la diversification de leurs portefeuilles. Certains d'entre eux sont notamment au courant des études récentes sur la surperformance des « sin stocks » (tabac, alcool etc..), ce qui renforce cette réticence à exclure certains secteurs particuliers. Nos gestionnaires de fonds seraient prêts à intégrer activement les critères ESG dans leur analyse financière, mais leur première préoccupation est que cela reste neutre envers la performance de leurs fonds. Ils voudraient tester historiquement («backtesting) l'effet d'une telle inclusion sur le passée afin de s'assurer que la performance de leurs fonds n'est pas impactée dans le futur.

Les facteurs écologiques et éthiques, ainsi que la gouvernance seraient les premiers critères à attirer leur attention. L'écologie et de l'éthique aurait un lien positif car ils réduisent le risque de réputation. La gouvernance les intéresse car les investisseurs institutionnels sont concernés par les droits des actionnaires minoritaires. Quelques-uns d'entre eux pensent que la réduction du risque de réputation et une bonne gouvernance dans le domaine de l'ESG pourrait créer la performance supplémentaire par rapport à leurs pairs. Le lien est très clair dans leur esprit entre les mauvaises nouvelles et une baisse des prix des actions de l'entreprise mais cela ne dure que pendant un certain temps seulement. Certain perçoivent aussi que l'analyse ESG a un coût pour l'entreprise, et ont tendance à croire que l'engagement dans l'ESG est neutre pour une entreprise sur le long terme, ces couts venant contrebalancer la réduction du nombre des mauvaises nouvelles .En résumé nos gestionnaires d'actifs semblent considérer que l'ESG réduit la volatilité et diminue le risque de réputation, mais a un impact neutre sur le long terme sur la performance.

En général, nos gestionnaires savent où trouver des données ESG et connaissent les grands fournisseurs de données et indice de références, notamment Bloomberg Transparence, Thomson Reuters Asset4 et s l'indice MSCI ESG. Ils mentionnent également un nombre croissant de fournisseurs de données ESG dans les pays nordiques. Il y a un consensus massif, cependant, sur le fait que les données ESG ne sont pas toujours vérifiées et mises à jour régulièrement, et si elles peuvent être utile comme un premier filtre, la plupart d'entre eux refont ou referait leur propre analyse. Ils estiment que cette analyse supplémentaire est très coûteuse, 
c'est pourquoi à l'heure actuelle, ils n'incluent les facteurs ESG dans leur analyse qu'au cas par cas, de manière à réduire un risque particulier.

Enfin, les gestionnaires reconnaissent tous que le facteur principal les poussant s'engager activement dans l'analyse ESG, au-delà d'une exclusion marginale, serait la demande expresse de clients. En deuxième position, une nouvelle réglementation dans les pays de leurs clients qui réglementerait les investissements serait un puissant moteur. Enfin, leur propre philanthropie pourrait être un élément déclencheur, si on peut leur prouver que cela est neutre pour la performance de leur portefeuille - notion très forte de préservation de la valeur pour le client.

\section{Conclusion}

Nous avons montré que le lien positif entre la performance des entreprises et leur engagement RSE n'est prouvé et perçu que dans certain contextes. D'autre part, il semblerait que les gestionnaires d'actifs soient prêts à intégrer des critères ESG seulement s'il est prouvé que cela est neutre pour la performance de leurs portefeuilles. Ils ne vont pas se livrer à ce type d'activité à moins d'une demande spécifique de leurs clients, car ils estiment que l'analyse ESG est très coûteuse pour eux, tout en n'ayant pas un impact significatif sur la performance du marché d'une entreprise, mais seulement sur le risque de réputation.

D'autre part, les investisseurs « traditionnels » soulignent la difficulté à évaluer la politique RSE des firmes, principalement due au manque d'information récente et pertinente dans ce domaine. Le coût additionnel ainsi lié à la nécessité de faire leur propre recherche crée une certaine barrière que tous ne veulent pas dépasser ou assumer compte tenu de l'absence de gain attendu sur la performance de leurs portefeuilles. 


\section{Bibliographie}

Albouy, 2005

Bénabou et Tirole (2006)

Belu (2009)

Chand (2006)

Cohen and Winn (2007)

Decock-Good, 1991

Friedman (1970)

Gössling, 2003

Maron (2006)

\section{Margolis \& Walsh (2003)}

Margolis, J., Elfenbein, H., Walsh, J., 2007, « Does it pay to be good ? A meta-analysis and rediretion of research on the relationship between corporate social and financial performance », Academy of Management Conference, Philadelphia.

McKinsey-BCCC (2008)

McGuire et al., 1988

Mackenzie et Lewis (1999)

Lewis et Mackenzie (1999)

Lewis et Mackenzie, 2000a

Lewis et Mackenzie (2000b)

Simon (1955)

Schubert and Lang (2005)

Van Beurden, P., Gössling, T., 2008, « The worth of values - A literature review on the relation between corporate social and financial performance », Journal ofBusiness Ethics, vol 82, pp. 407-424.

Waddock et Graves, 1997

Webley et al. (2001)

Wu, M.-L., 2006, « Corporate social performance, corporate financial performance, and firm size: A meta-analysis », Journal of American Academy of Business, vol 8, issue 1, pp. 163-171.

\section{Bibliographie}

Amenc, N., Le Sourd, V., 2008, « Socially responsible investment performance in France », EDHEC Risk and Asset Management Research Centre.

Bauer, R., Koedijk, K., Otten, R., 2005, « International evidence on ethical mutual fund performance and investment style », Journal of Banking \& Finance, vol. 29, issue 7, pp. 1751-1767.

Bauer, R., Derwall, J., Otten, R., 2006, « The ethical mutual fund performance debate : New evidence from Canada », Journal of Business Ethics (2007), vol 70, pp. 111-124.

Bauer, R., Otten, R., Tourani Rad, A., 2006, « Ethical investing in

Australia: Is there a financial penalty? » Pacific-Basin Finance Journal, vol 14 issue 1, pp. 33-48.

Bello, Z., 2005, " Socially responsible investing and portfolio diversification », Journal of Financial Research, vol 28, issue 1, pp. 41-57.

Brammer, S., Millington, A., 2008, « Does it pay to be different? An analysis of the relationship between corporate social and financial performance », Strategic Management Journal, vol 29, issue 12, pp. $1325-1343$.

Butz, C., 2003, « Decomposing SRI performance », Pictet Quants report. 
Clarkson, P., Fang, X., Li, Y., Richardson, G., 2010, « The relevance of environmental disclosures for investors and other stakeholder groups : Are such disclosures incrementally informative?»

Clow, J. E., 1999, « Money that grows on trees », Institutional Investor, vol 33, issue 10, pp. 212-215.

Cordeiro, J. J., Sarkis, J., 1998, « Environmental proactivism and firm performance : evidence from security analyst earnings forecasts », Business Strategy and the Environment, vol 6, issue 2, pp. 104-114.

Cormier, D., Magnan, M., 2007, « The revisited contribution of environmental reporting to investors' valuation of firm's earnings : An international perspective », Ecological Economics, vol 62, issue 3-4, pp. 613-626.

Core, J. E., Guay, W. R., Rusticus, T. O., 2006, « Does weak governance cause weak stock returns? An examination of firm operating performance ans investors expectations », The Journal of Finance, vol 61, issue 2, pp. 655-687.

Cummings, L. S., 2000, «The financial performance of ethical investment trusts : An australian perspective », vol 25, issue 1, pp. 79-92.

D'Antonio, L., 2000, « Socially responsible investing and asset allocation », The Journal of Investing, vol 9, No 3, pp. 65-72.

Derwall, J., Guenster N., Bauer, R., Koedijk, K., 2005, « The eco-efficiency premium puzzle », Financial Analyst Journal. Vol. 61, No 2.

Derwall, J., Koedijk, K., 2009, « Socially responsible fixed-income funds », Journal of Business Finance \& Accounting, 36(1) \& (2), 210-229, January/March 2009.

De Klerk, M., De Villiers, C., 2012, «The value relevance of corporate responsibility reporting: South African evidence», Meditari Accountancy Research, vol 20, Issue 1, pp.21-38.

Di Vita, G., 2009, « Legal families and environmental protection : Is there a causal relationship ? », Journal of Policy Modeling, vol 31, No 5, pp. 694-707.

Earnhart, D., Lizal, L., 2007a, « Effect of pullution control on dorporate financial performance in transition economy », European Environment, vol 17, pp. 247-266.

Earnhart, D., Lizal, L., 2007b, « Does better environmental performance affect revenues, cost, or both ? Evidence from a transition economy », William Davidson Institute, working paper number 856.

Eccles R. G., Ioannou I., Serafeim G., 2012, « The Impact of a corporate culture of sustainability on corporate behavior and performance », NBER Working paper No. 17950.

Fernandez-Izquierdo, A., Matallin-Saez, J., C., 2008, « Performance of ethical mutual funds in Spain : Sacrifice or premium ? », Journal of Business Ethics, vol 81, pp. 247-260.

Galema, R., Plantinga, A., Scholtens, B., 2008, « The stocks at stake : Return and risk in socially responsible investment », Journal of Banking \& Finance.

Gillet, P., 2008, « Les performances des fonds « éthiques » et vicieux », Etats Généraux du Management, Paris, octobre.

Goldreyer, E., Ahmed, P., Diltz, J., 1999, « The performance of socially responsible mutual funds: Incorporating sociopolitical information », Journal of Financial Research, vol 28, pp. 41-57.

Gompers, P., Ishii, J., Metrick, A., 2003, « Corporate governance and equity prices », Quarterly Journal of Economics, vol. 118, issue 1, pp. 107-156.

Geczy, C.C., Stambaugh, R.F., Levin, D., 2003, « Investing in socially responsible mutual funds », Working Paper Wharton School.

Gregory, A., Whittaker, J., 2007, « Performance and performance persistence of 'ethical' unit trusts in the UK », Journal of Business Finance \& Accounting, vol 34, issue 7-8, pp. 1327-1344.

Hamilton, S., J, H., Statman, M., 1993, Doing well while doing good? The investment performance of socially responsible mutual funds », Financial Analysts Journal, vol 49, issue 6, pp. 62-66.

Hillman, T., Keim, G. D., 2001, « Shareholder value, stakeholder management, and social issues : What's the bottom line? », Strategic Management Journal, vol. 22, No 2, pp. 125-139.

Jaggi, B., Freedman, M., 1992, «An examination of the impact of pollution performance on economic and market performance : Pulp and paper firms », Journal of Business Finance \& Accounting, vol 19, issue 5, pp. 697-713. 
Kempf, A., Osthoff, P., 2007, « The effect of socially responsible investing on portfolio performance », European Financial Management, vol 13, issue 5, pp. 908-922.

King, A., A., Lenox, M., J., 2001, « Does it really pay to be green ? An empirical study of firm environmental and financial performance », Journal of Industrial Ecology, vol 5, issue 1.

Konar, S., Cohen, M. A., 2001, « Does the market value environmental performance ? », Review of Economics \& Statistics, vol 83, issue 2, pp. 281-289.

Luther, R., Matatko, J., 1994, "The performance of ethical unit trusts: Choosing an appropriate benchmark » British Accounting Review, vol 26, pp. 77-89.

Luther, R., Matatko, J., Corner, D., 1992, "The investment performance of UK ethical unit trusts », Accounting, Auditing and Accountability Journal Review, vol 5, pp. 57-70.

Mallin, C.A., Saadouni, B., Briston, R.J., 1995, « The financial performance of ethical investment funds », Journal of Business Finance and Accounting, vol 22, issue 4, pp. 483-496.

Margolis, J., Elfenbein, H., Walsh, J., 2007, « Does it pay to be good? A meta-analysis and rediretion of research on the relationship between corporate social and financial performance ", Academy of Management Conference, Philadelphia.

Mattingly, J. E., Berman, S., 2006, « Measurment of corporate social action discovering taxonomy in the Kinder Lydenburg Domini ratings data », Business \& Society, vol 45, pp. 20-46.

Nakao, Y., Amano, A., Matsumura, K., Genba, K., Nakano, M., 2007, " Relationship between environmental performance and financial performance : an empirical analysis of japanese corporations », Business Strategy \& the Environment, vol 16, issue 2, pp. 106-118.

Okamoto, D., 2009, « Social relationship of firm and the CSP-CFP relationship in Japan : Using artificial neutral networks », Journal of Business Ethics, vol 87, pp. 117-132.

Renneboog, L., Horst, J., T., Zhang, C., 2008, « The price of ethics and stakeholder governance : The performance of socially responsible mutual funds », Journal of Corporate Finance, vol 14, pp. 302-322.

Russo, M., V., Fouts, P., A., 1997, « A resource-based perspective on corporate environmental performance and profitability », Academy of Management Journal, vol 40, issue 3, pp. 534-559.

Schroder, M., 2004, «The performance of socially responsible investments: investment funds and indices », Financial Markets and Portfolio Management, vol 18, issue 2, pp. 122-142.

Saadaoui, K., 2012, " The financial performance and investment style of french ethical funds », 29th International Conference of the French Finance Association (AFFI) 2012.

Scholtens, B., 2005, « What drives socially responsible investment? The case of Netherlands », Wiley InterScience (www.interscience.wiley.com)

Stanwick, P.A., Stanwick, S.D., 1998, « The relationship between corporate social performance and size, financial and environmental performance », Journal of Business Ethics, vol 17, issue 2, pp. 195-204.

Statman, M., 2000, « Socially responsible mutual funds », Financial Analysts Journal, vol 56, issue 3, pp. 30-39.

Von Arx, U., Ziegler, A., 2008, « The effect of CSR on stock Performance : New evidence for the USA and Europe », CER-ETH - Center of Economic Research at ETH Zürich, Working Paper No 08/85.

Van Beurden, P., Gössling, T., 2008, « The worth of values - A literature review on the relation between corporate social and financial performance », Journal ofBusiness Ethics, vol 82, pp. 407-424.

Wagner, M., 2001. « A review of empirical studies concerning the relationship between environmental and financial performance. What does the evidence tell us?. Center

for Sustainability Management, vol 52.

Wilcox, W., Berry, K., O’Bryan, D., Quirin, J., J., 2010, « The relevance of discretionary disclosures : Predictive value versus feedback value », Journal of Business \& Economics Research, vol 8, No 3.

Wu, M.-L., 2006, « Corporate social performance, corporate financial performance, and firm size: A meta-analysis », Journal of American Academy of Business, vol 8, issue 1, pp. 163-171. 
Ziegler, A., Schröder, M., Rennings, K., 2007, « The effect of environmental and social performance on the stock performance of european corporations », Environmental and Ressource Economics, vol 37, issue 4, pp. 661-680.

Appendix A

Questionnaire ISR (Investissement Socialement Responsable) pour les gestionnaires de fonds Suisses :

- Que pensez-vous en général de l'engagement des entreprises Suisses en termes de développement durable?

- Avez-vous déjà inclus dans vos décisions d'investissement des critères ESG, c'est à dire environnementaux, sociaux/éthiques ou de gouvernance d'entreprise, ou exclu certain secteurs (alcool, tabac) dans la sélection d'actions Suisses? Pour d'autres pays?

- Pensez-vous qu'il existe un lien entre la performance (boursière ou financière) d'une entreprise et ses effort en terme de :

-gouvernance d'entreprise

-écologie

-sociétal / éthique (travail des enfants, esclavage etc...)

-social (interne à l'entreprise, sa politique sociale, partage t'-elle ses bénéfices avec ses employés par exemple ?)

- Connaissez -vous des fournisseurs de données pour juger les entreprises Suisses ou internationales selon les critères ESG ?

* Pensez-vous que ces informations soient crédibles?

* Les utilisez-vous et dans quel cadre?

- Qu'est-ce qui pourrait vous pousser à considérer de tels critères dans votre gestion ? (demande de client, étude scientifique, régulation...) 